Recherches amérindiennes au Québec

\title{
Cooptation et résistance dans la planification forestière concertée au Québec
}

Le cas des Atikamekw Nehirowisiwok et des " tables GIRT »

Cooptation and resistance in collaborative forest planning in Québec

The case of Atikamekw Nehirowisiw and the "Tables GIRT"

\section{Cooptación y resistencia en la planificación forestal concertada en Québec}

El caso de los Atikamekw Nehirowisiwok y de las mesas de gestión "GIRT"

E witapimakaniwitcik atikamekw nehirowisiwok aski e nisawitakaniwok okiskeritamowiniwaw e kicteritakonik acitc aniki « GIRT » ka icinikatakaniwitcik

Jean-François Fortier et Stephen Wyatt

Volume 44, numéro 1, 2014

Les Atikamekw Nehirowisiwok : territorialités et savoirs

URI : https://id.erudit.org/iderudit/1027878ar

DOI : https://doi.org/10.7202/1027878ar

Aller au sommaire du numéro

Éditeur(s)

Recherches amérindiennes au Québec

ISSN

0318-4137 (imprimé)

1923-5151 (numérique)

Découvrir la revue

Citer cet article

Fortier, J.-F. \& Wyatt, S. (2014). Cooptation et résistance dans la planification forestière concertée au Québec : le cas des Atikamekw Nehirowisiwok et des « tables GIRT ». Recherches amérindiennes au Québec, 44(1), 35-47. https://doi.org/10.7202/1027878ar

\section{Résumé de l'article}

Définies comme des processus de concertation où les utilisateurs du territoire sont invités à élaborer une vision commune de la gestion des territoires forestiers, les tables de gestion intégrée des ressources et du territoire (« tables GIRT ») sont apparues au Québec en 2010. Cet article explore la participation des Atikamekw Nehirowisiwok à ces tables, et contrairement à d'autres études qui tendent à interpréter les processus collaboratifs comme un modèle idéal " gagnant-gagnant " ou comme une stratégie de cooptation de la participation autochtone, les auteurs proposent ici d'examiner les processus de concertation comme un lieu où se manifestent à la fois des formes de résistance et de cooptation. De plus, les résultats démontrent que la participation autochtone aux tables GIRT gagne à être comprise en tenant compte des autres processus dans lesquels les Premières Nations sont également engagées. 


\section{Jean-François Fortier}

Département de sociologie,

Université Laval,

Québec

et

\section{Stephen Wyatt}

Faculté de

foresterie,

Université de

Moncton,

Nouveau-Brunswick

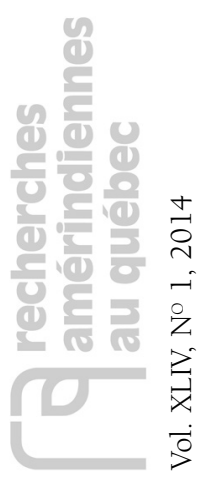

$A^{\mathrm{U}}$

u COURS DE L'ANNÉE 2010, les tables locales de gestion intégrée des ressources naturelles et du territoire (ci-après « tables GIRT ») sont apparues un peu partout à travers le Québec. Ces tables peuvent être définies comme des processus de concertation à travers lesquels les utilisateurs d'un territoire délimité déterminent conjointement les « objectifs locaux d'aménagement durable des forêts». Les tables GIRT ont été implantées avant tout pour répondre aux exigences légales de la nouvelle Loi sur l'aménagement durable du territoire (L.R.Q. c. A-18.1). Ladoption de cette loi entraîne notamment le transfert, de l'industrie forestière à l'État, de la responsabilité de la planification forestière, soit plus spécifiquement les directions générales régionales du ministère des Forêts, de la Faune et des Parcs du Québec (MFFPQ²). Ainsi, les tables GIRT sont amenées à collaborer avec l'État dans le but de reconnaître les besoins et les préoccupations des intervenants et des utilisateurs du territoire lors de l'élaboration des plans d'aménagement forestier intégré (PAFI).

Les tables GIRT ne sont pas les premiers processus de concertation à voir le jour dans le milieu forestier au Québec (Martineau-Delisle 2013; Leclerc et Andrew 2013; Théberge 2012; Nadeau et al. 2004; Côté et Bouthillier 2002). Par exemple, au début des années 1990, dans la région de la Mauricie, une table de concertation sur le développement durable a été mise en place afin d'élaborer une vision intégrée du territoire en réunissant des intervenants des secteurs récréatifs, fauniques et forestiers (Burquel 1992). Cette table constituait un projet pilote mené à l'intérieur d'une « aire commune » dont le responsable de la planification et de l'aménagement forestier était la compagnie Stone-Consolidated (ibid.). Des comités de concertation ont aussi vu le jour au tournant des années 2000 dans la foulée des premières démarches de certification ISO 140001 et CSA Z808 (Côté et Bouthillier 2002).

Assistant à l'apparition de ce type de mécanisme un peu partout au Canada, Smith et Bombay écrivaient au milieu des années 1990 que ce modèle abordait la participation autochtone «seulement comme une valeur de plus dans un large éventail d'intérêts concurrents » (1995: 5). Les Premières Nations ont critiqué aussitôt cette position en arguant que cela pouvait mener à l'extinction des droits autochtones et du titre aborigène (Smith et Bombay 1995 : 6). Par ailleurs, la Commission royale sur les peuples autochtones recommandait en 1992 de considérer dorénavant les peuples autochtones comme des «nations» avec tous les droits et responsabilités que cela impliquait: «Ainsi, les peuples autochtones ne doivent pas être traités simplement comme un "autre intervenant" dans les négociations liées aux ressources [naturelles]. » (McGregor 2011 : 301) 
Smith et Bombay soutiennent néanmoins que les autochtones « ont beaucoup à gagner en participant » (Smith et Bombay 1995 : 11) puisque ces processus permettraient de fournir une tribune pour sensibiliser le public aux questions et valeurs autochtones, de développer à court terme des mesures d'atténuation environnementale et de créer des alliances avec d'autres groupes afin d'influencer les décisions entourant l'utilisation des ressources et du territoire (ibid.).

D'une part, contrairement à d'autres études qui tendent à interpréter les processus collaboratifs soit comme un modèle idéal « gagnant-gagnant» ou comme une forme de cooptation de la participation autochtone dans la gestion des forêts, cet article propose d'examiner comment les processus de concertation, tels que les tables GIRT, représentent un lieu où se manifestent à la fois des formes de résistance et d'affirmation, de cooptation et de collaboration (Takeda et Røpke 2010; Howlett et al. 2009; Spaeder et Feit 2005; Feit 2005). D'autre part, à partir du cas des Atikamekw Nehirowisiwok, cette étude démontre que la participation autochtone dans les tables GIRT gagne à être comprise en tenant compte des autres processus dans lesquels les Premières Nations sont également engagées (p. ex. négociation territoriale avec les gouvernements, consultations distinctes, désobéissance civile, recours judiciaires, etc.).

Larticle débute par une mise en contexte de la participation autochtone dans la gestion forestière au Canada, suivie par la présentation du cadre méthodologique. Le processus de planification forestière est ensuite examiné afin de situer le cadre dans lequel ces nouveaux modes de concertation s'inscrivent. Il est maintenant bien connu que ces processus sont plus souvent qu'autrement le sousproduit d'un cadre préexistant (Nadasdy 2003; Natcher et Davis 2007). Larticle s'enchaîne avec la présentation des résultats et se termine avec une discussion sur les résultats.

\section{Les Premières Nations et la gestion forestière AU CANAdA : DE L'EXCLUSION À LA COEXISTENCE}

Il est maintenant assez bien admis qu'il existe un « parallèle très étroit entre l'exploitation des forêts et la colonisation des peuples autochtones » (McGregor 2006). Comme le soulignait McGregor, « à mesure que les nouveaux arrivants européens dominaient progressivement les territoires des peuples autochtones, ces derniers se trouvaient de plus en plus coupés des territoires, souvent forestiers » (ibid. : 62). Maintes études ont illustré comment les gouvernements provinciaux qui se sont vu conférer la gestion et le développement des «terres publiques » sous la Loi constitutionnelle de 1867 ont pris des décisions et ont adopté des politiques sans vraiment tenir compte de l'occupation du territoire par les Premières Nations (McGregor 2006; Stevenson et Webb 2003; Simard 2003; Gélinas 2002; McNeil 1997; Savard 1981). Par contre, certains auteurs remettent en question cette conception selon laquelle les Premières Nations sont demeurées passives devant ce mouvement de colonisation et de développement (voir, entre autres, Gélinas 2003; Poirier 2000).
Par exemple, «plutôt que de subir passivement le commerce des fourrures, [les autochtones] ont grandement contribué à en définir la forme » (Gélinas 2003 : 16). Néanmoins, on peut raisonnablement argumenter que la création des « réserves indiennes » et le développement du territoire (p. ex. par l'octroi des permis de coupe aux industries forestières, la création des réservoirs d'eau et l'implantation des centrales hydroélectriques) ont limité progressivement l'accès au territoire ancestral par les Premières Nations.

Lémergence d'une conception « durable » du développement a contribué à reconnaître l'importance de la participation des peuples autochtones à la gestion du territoire. La Déclaration de Rio (Nations unies 1992a) sur le développement et l'environnement ainsi que la Déclaration de principe sur les forêts reconnaissent un rôle essentiel aux Premières Nations dans la gestion des forêts afin d'assurer un «développement durable» du territoire et des ressources. Le principe 5 de la Déclaration invite les gouvernements à s'assurer que leurs "politiques forestières » rendent compte des intérêts et des besoins des autochtones (Nations unies 1992b). De plus, le chapitre 26 de l'Agenda 21 souligne que les populations autochtones et leurs communautés devront exercer « un plus grand contrôle sur leurs terres, gérer de façon plus autonome leurs ressources et prendre une part accrue aux décisions en matière de développement qui les concernent » (Nations unies 1992c). LAgenda 21 encourage aussi les États nationaux à implanter des processus de consultation « appropriés » afin d'intégrer leurs "valeurs », leurs " besoins » ainsi que leur «savoir-faire traditionnel » dans les processus de gestion et de développement, et ce tant au niveau « national » que « régional » (ibid.).

Bien que les principes énoncés n'aient aucun poids juridique, plusieurs États nationaux ont posé des actions en réponse à cette déclaration. À ce titre, en 1995, le Conseil canadien des ministres des forêts (CCMF) a élaboré une première série de critères et d'indicateurs sur l'aménagement forestier durable dont la dernière mise à jour remonte à plus de dix ans (CCMF 1995 et 2003). Le CCMF a voulu faire reconnaître et respecter les droits des Premières Nations ainsi que leurs valeurs et l'utilisation qu'elles font de la forêt. Il a précisé notamment que « les plans d'aménagement forestier doivent refléter les options examinées et les mesures prises concernant les droits ancestraux et issus de traités » (CCMF 2003 : 18).

Plus particulièrement au Québec, les Cris et le gouvernement du Québec ont signé en 2002 une Entente concernant une nouvelle relation appelée communément la « Paix des braves $»$. Cette entente représente une des avancées les plus prometteuses pour redonner une place significative aux Premières Nations dans la gestion forestière au Québec. Cette entente prévoit notamment la mise en place d'un « régime forestier adapté » dont la création du Conseil Cris-Québec sur la foresterie (CCQF) vise «à permettre une consultation étroite des Cris lors des différentes étapes de planification et de gestion des activités forestières afin de mettre en ceuvre le régime forestier adapté » 
(Québec 2002). Alors que certains demeurent sceptiques face à ce type d'entente (Natcher et Davis 2007; Rodon 2003; Feit et Beaulieu 2001), pour d'autres elle illustre un virage significatif vers une gouvernance « partagée» du territoire où «les droits et les intérêts des Autochtones constituent maintenant une variable incontournable à laquelle l'État doit accorder une attention particulière » (Grammond 2009 : 946).

Depuis les arrêts Taku River (2004) et Haïda (2004), l'État se retrouve dans l'obligation de consulter et d'accommoder les Premières Nations sur tout projet ou décision susceptible d'avoir un impact sur leur titre ou leur droit, et ce, sans que ces dernières n'aient encore prouvé leurs titres ou leurs droits ancestraux (Première Nation Tinglit de Taku River c. C.-B. [2004]). Cette nouvelle obligation prend un sens encore plus important au Québec puisque la majorité des nations autochtones n'ont pas encore cédé, ni fait reconnaître leurs titres et leurs droits ancestraux (Grammond 2009). En fait, seuls les Cris, les Inuits et les Naskapis ont cédé leur titre et leurs droits ancestraux en échange de droits spécifiques en signant la Convention de la Baie James et du Nord-Est québécois (SAA 1998). Il n'est donc pas surprenant de constater l'adoption d'un Guide intérimaire en matière de consultation des communautés autoch-

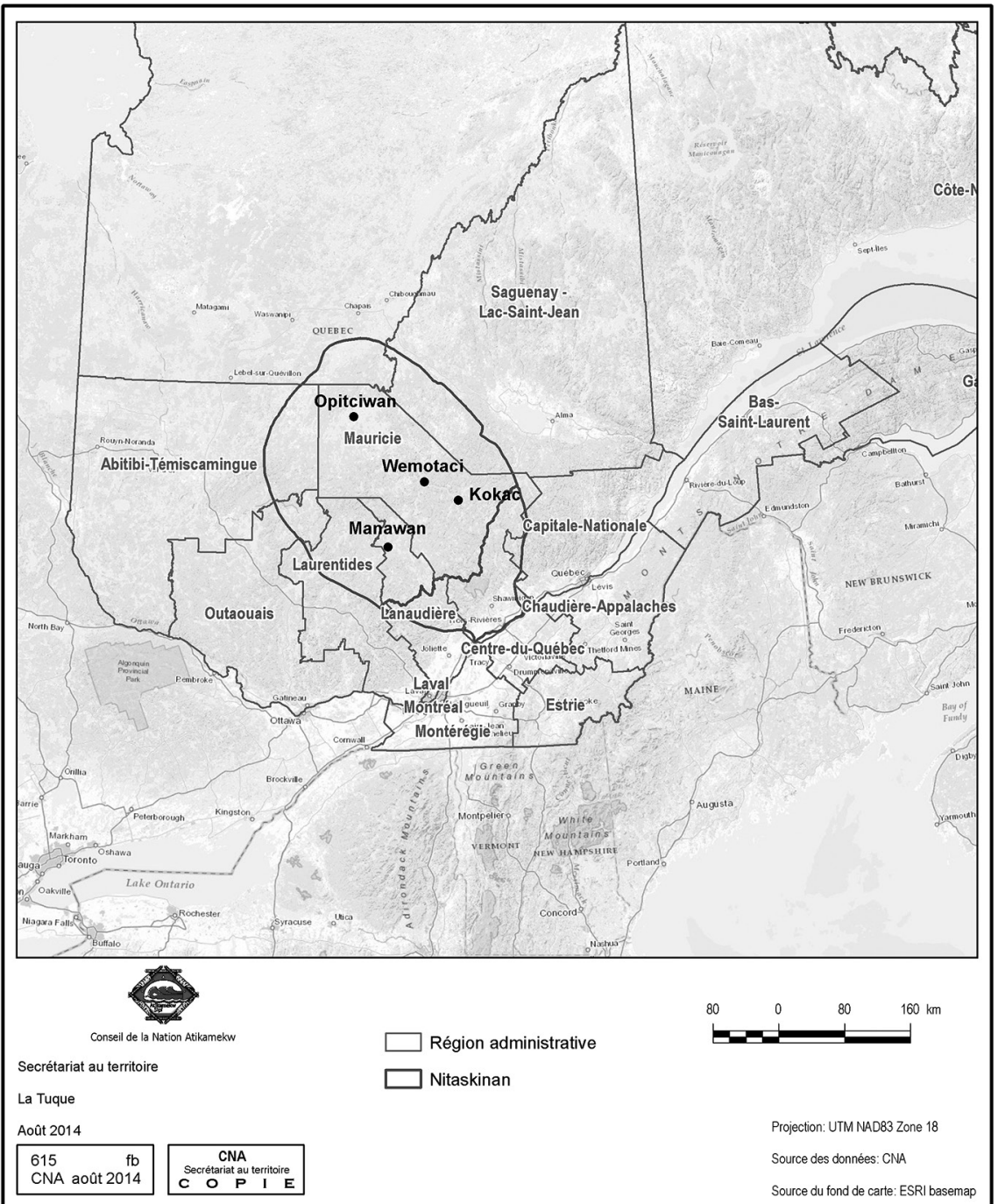

Localisation des communautés d'Opitciwan, de Wemotaci et de Manawan, ainsi que de la réserve inhabitée (" Kokac ") du Nitaskinan et des régions administratives du Québec

(Source : Conseil de la Nation atikamekw 2014) tones par le gouvernement du Québec

afin de mieux baliser cette nouvelle responsabilité (Québec 2008). Selon Grammond, les arrêts Haïda et Taku River ont élargi le modèle de "gouvernance territoriale partagée » établi dans les traités comme ceux de la CBJNQ ou de la Paix des braves, à l'ensemble des Premières Nations qui revendiquent un titre et des droits ancestraux à l'échelle du pays (Grammond 2009 : 948).

Ce que certains appellent la « coexistence » devient en quelque sorte le point de référence pour évaluer la place des Premières Nations dans la foresterie (McGregor 2011; Stevenson et Natcher 2010; Wyatt 2004; Stevenson et Webb 2003). Lidée de coexistence renvoie à une situation où les cultures autochtones et non autochtones continuent de s'épanouir côte à côte en se supportant mutuellement mais sans que l'une domine l'autre. Selon McGregor: «Elle est fondée sur la croyance que d'avoir différentes visions du monde ne doit pas être une chose indésirable et que l'élaboration d'un cadre qui respecte les différentes visions du monde serait une approche positive à prendre. » (2011 : 307) Un des exemples les plus éloquents d'une telle coexistence est celui du wampum à deux voies d'Haudenosaunee ( colliers de traité ») qui ont des couleurs différentes se prolongeant côte à côte, séparées par une troisième bande d'une autre couleur (Ransom et Ettenger, cités dans McGregor 2011). Le wampum à deux voies peut très bien servir à nouveau de modèle pour renouveler et renégocier la relation entre les deux solitudes, comme le suggère McGregor (2011 : 307).

Cet idéal de coexistence entre des nations « distinctes » et « égales » implique d'arrêter de concevoir les Premières Nations au même titre que les autres « intervenants » ou « utilisateurs » afin de reconnaître notamment leurs droits qui se distinguent de ceux des autres citoyens canadiens, tel que souligné par la RCAP et dans les déclarations internationales comme celles de l'ONU mentionnées précédemment. Il s'agit également ici de respect des demandes des Premières Nations qui exigent de plus en plus le contrôle de la gestion des terres ancestrales, ou du moins une forme de cogestion qui leur permettrait de jouer un rôle à titre de partenaire égal dans un esprit de coexistence. La 
récente déclaration de souveraineté par les Atikamekw Nehirowisiwok sur leur territoire ancestral (voir carte) qu'ils nomment le «Nitaskinan» ( notre territoire») illustre très bien cela (Robillard 2014). À ce titre, ceux-ci ont clairement précisé qu'ils n'étaient pas contre le développement, mais plutôt qu'ils n'accepteraient plus aucune forme de développement sur le Nitaskinan sans leur consentement préalable, libre et éclairé. La prochaine section présente le cadre méthodologique ayant servi à mener une étude de cas sur la participation des Nehirowisiwok à la planification forestière par l'entremise des tables GIRT.

\section{LA PARTICIPATION NEHIROWISIW AUX TABLES GIRT}

Les Atikamekw Nehirowisiwok représentent l'une des dix Premières Nations présentes au Québec en plus des Nunavimmiut («Inuits»). Appelés autrefois « Têtes-deBoule » ou « Attikamègues », et aujourd'hui « Atikamekw », ils s'autodésignent en tant que «Nehirowisiwok» (« Nehirowisiw» au singulier). La population nehirowisiw compte plus de 6700 membres répartis dans trois communautés (15\% d'entre eux vivent à l'extérieur des « réserves »): Opitciwan et Wemotaci étant situées dans la Haute-Mauricie, et Manawan dans la région de Lanaudière. La majorité des Nehirowisiwok parlent couramment l'atikamekw et utilisent le français comme langue seconde. Chaque communauté est administrée par un conseil de bande élu par suffrage universel. On retrouve également le Conseil de la Nation atikamekw (CNA) composé des trois chefs de bande et d'un Grand Chef élu. Le CNA a le mandat de mener les négociations territoriales auprès des gouvernements et offre divers services, notamment dans les domaines de l'éducation, de la culture, de l'emploi, de la santé et des services sociaux.

Vers la fin $\mathrm{du} \mathrm{XIX}^{\mathrm{e}}$ siècle, faisant face à une pénurie des ressources fauniques et à des pressions de développement, dont l'exploitation forestière, les Nehirowisiwok formuleront une première série de demandes en matière de gestion territoriale, incluant entre autres la création des réserves afin de "gérer plus efficacement une portion de leur territoire d'exploitation et les ressources animales qui s'y trouvaient» (Gélinas 2002: 39). Toutefois, selon Gélinas, la création des réserves n'a pas permis aux Nehirowisiwok de « se mesurer au mouvement de colonisation eurocanadien $»$ (ibid. : 45). L'exploitation forestière pratiquée initialement sur le territoire de la Mauricie était peu mécanisée et certains Nehirowisiwok travaillaient à titre de bûcherons ou de draveurs (Gosselin 2004). À partir des années 1980, les Nehirowisiwok se sont dotés de plusieurs organisations afin de faire reconnaître leur occupation du territoire et de renforcer leur participation à la gestion des territoires forestiers. Cela débute en 1982 alors qu'un service forestier est créé avec la fondation du CNA (ibid.). En 1990, l'Association Mamo Atoskewin Atikamekw (AMAA) est mise sur pied afin de défendre et de représenter les intérêts des trappeurs et chasseurs nehirowisiwok (Houde 2012; Wyatt 2004 et 2006). En 1992, Manawan et Wemotaci fondent ensemble les Services forestiers Atikamekw Aski inc. (SFAA). En 1996, le
Conseil des Atikamekw de Wemotaci conclut une entente avec une entreprise forestière afin de mettre sur pied une scierie : « Tackipotcikan » (qui signifie « outil pour couper du bois »). Toutefois, le projet sera mis sur la glace en 2001 à cause du conflit du bois d'œuvre canado-américain. En 1999, un « projet d'harmonisation » est mis en branle par le SFAA et le Conseil des Atikamekw de Wemotaci. Le projet vise à élaborer une nouvelle approche afin de concilier les activités traditionnelles des Nehirowisiwok et le développement forestier. Selon Wyatt, cette approche représente une des avancées les plus importantes de la participation nehirowisiw dans la gestion forestière (Wyatt 2004 et 2006). Par ailleurs, le Conseil des Atikamekw d'Opitciwan gère depuis 1999 une scierie en copartenariat avec une entreprise forestière dont il détient la majorité des parts (55/45). Plus récemment, les Nehirowisiwok ont été invités à participer à des processus de planification forestière concertée appelés communément « tables locales de gestion intégrée des ressources et du territoire ». Ainsi, cette étude de cas se penche sur les développements récents de la participation des Nehirowisiwok à la planification forestière par l'entremise de ces nouveaux modes de concertation.

Les études antérieures et actuelles (Wyatt et al. 2010; Wyatt 2004; Fortier, en préparation) indiquent que les Nehirowisiwok prennent part également à d'autres processus qui leur permettent déjà d'être consultés lors de la confection des plans d'aménagement forestier. Par conséquent, pourquoi les Nehirowisiwok participeraient-ils aux tables GIRT? On se questionne ainsi à savoir quelle est la nature de leur participation aux tables GIRT et comment celle-ci influence-t-elle les travaux des tables dans le cadre de la planification et de l'aménagement des territoires forestiers? Il ne s'agit donc pas tant d'examiner les effets des tables GIRT sur la planification forestière mais plutôt les caractéristiques ainsi que les retombées de la participation nehirowisiw aux tables GIRT. Cinq objectifs ont été fixés pour répondre à ces questions: (a) cerner le ou les objectifs ainsi que le mode de fonctionnement des tables GIRT; (b) identifier les participants nehirowisiwok aux tables GIRT, de 2010 à 2013; (c) décrire le ou les rôle(s) exercé(s) par eux au sein des tables, de 2010 à 2013; (d) évaluer les impacts de leur contribution de 2010 à 2013; et (e) identifier, s'il y a lieu, les changements dans cette participation depuis la mise sur pied des tables GIRT en 2010, afin de mieux comprendre leur dynamique dans le temps ainsi que les principaux facteurs à l'origine de ces changements.

Létude de cas a été retenue comme méthode pour examiner la participation des Nehirowisiwok dans la planification forestière. L'étude de cas forme un « sous-système » représentatif d'un « système plus large » qui tient compte de son « contexte immédiat, son histoire et ses différentes dimensions » (Roy 2010: 207). Il s'agit d'une enquête qualitative exploratoire basée sur une pluralité de sources de données dont l'une des principales stratégies d'analyse consiste à trianguler les différentes sources de données. Pour délimiter la collecte de données, la participation 
nehirowisiw a été analysée dans les tables GIRT de Lanaudière (Table GIRT 062) et de la Mauricie (Tables GIRT 026-51, 041-51, 042-51, 043-51 et 043-52).

Plus d'une douzaine d'entretiens ont été réalisés auprès des participants nehirowisiwok ainsi que des responsables des tables. Les personnes rencontrées représentent diverses organisations, dont les Commissions régionales des ressources naturelles et du territoire (CRRNT) de la Mauricie et de Lanaudière, la Direction générale de la Mauricie et du Centre-du-Québec, l'Unité de gestion Windigo et Gouin du MFFPQ ainsi que les Conseils des Atikamekw de Manawan, de Wemotaci et d'Opitciwan. Les entretiens ont permis notamment de documenter le point de vue des participants sur le mode de fonctionnement des tables GIRT, le rôle des Nehirowisiwok et les impacts de leur participation. Certains facteurs qui ont influencé leurs pratiques ont aussi été abordés lors des entretiens.

Les données recueillies proviennent également d'une recherche documentaire. Linformation amassée comprend des comptes rendus de rencontres, des rapports d'activités, des sites Internet, des pamphlets, des publications gouvernementales, des articles de journaux et ainsi de suite. Des demandes particulières ont également été adressées directement aux CRRNT de la Mauricie et de Lanaudière afin de compléter la collecte.

Lobservation non participante a été utilisée pour examiner les interventions des membres nehirowisiwok au sein d'une table GIRT en temps réel, ce qu'il n'est pas possible de faire avec la recherche documentaire ou les entretiens. Au total, une seule séance d'observation a été menée lors de la rencontre du 23 octobre 2013 de la Table GIRT 062.

L'examen du mode de fonctionnement des tables GIRT visait à délimiter le cadre participatif à partir d'une description du mandat, de la composition ainsi que des règles et modalités de fonctionnement (p. ex. processus de nomination des membres et mode de prise de décisions). Cet examen a été réalisé essentiellement à partir d'une analyse de la documentation pertinente et disponible à cet effet telle que les Règles et modalités de fonctionnement des tables de gestion intégrée des ressources et du territoire (CREM 2013). Les entretiens ont également permis de compléter la description en plus de valider comment ces tables fonctionnent en pratique.

Létude de la participation nehirowisiw est avant tout exploratoire. Bien que certaines hypothèses aient guidé l'analyse, les pratiques participatives des Nehirowisiwok ont été examinées sous trois angles: qui participe chez les Nehirowisiwok (Plummer et FitzGibbon 2004), comment ils participent (von der Porten et de Loë 2014) et quels sont les impacts de cette participation (Conley et Moote 2003; Gunton et Day 2003). Le « qui » est important afin de vérifier qui participe réellement et quels sont les groupes et les organisations qui sont représentés dans ces processus. Un portrait des participants nehirowisiwok et de leur représentativité au sein des tables GIRT (Nehirowisiwok / non Nehirowisiwok) a donc été réalisé à partir des données collectées.
La description du « comment » a été réalisée principalement à partir des commentaires recueillis dans les entretiens et des comptes rendus des rencontres. Elle vise à examiner le rôle des Nehirowisiwok à la lueur de ce qui a été discuté dans la mise en contexte (" nation » vs « intervenant» ou « utilisateur »). De plus, la fréquence de la participation nehirowisiw aux rencontres des tables GIRT a été utilisée pour évaluer l'étendue de la présence nehirowisiw à ces rencontres, ce qui nous paraît essentiel pour dresser un premier portrait de leur participation. Il s'agit de vérifier s'ils sont bel et bien présents ou non aux rencontres (et non pas seulement sur papier). Cela permet ensuite de poser la question de savoir ce qui expliquerait une présence soutenue, sporadique ou faible de la part du participant nehirowisiw et, s'il y a lieu, les variations observées. La fréquence de la participation a été établie à partir des listes des présences que l'on retrouve dans les comptes rendus des rencontres. Enfin, les répondants à l'étude ont été questionnés afin d'identifier quels étaient selon eux les principaux impacts de la participation nehirowisiw, et ce, tant du point de vue des coordonnateurs des tables GIRT (CRRNT et l'État) que du point de vue des Nehirowisiwok. Nous reconnaissons toutefois que d'autres études ont souligné les limites d'une telle approche (Diduck et Sinclair 2002).

Enfin, la sous-dimension temps est également importante puisque la participation nehirowisiw aux tables GIRT est appréhendée comme étant dynamique, et non statique (Carlsson et Berkes 2005; Pinkerton 2003). Ainsi, nous avons tenté de vérifier si leur participation aux tables GIRT a fluctué dans le temps et s'il y a eu des changements. Nous avons aussi essayé de cerner les facteurs qui expliqueraient les mouvances observées. Les changements et les facteurs ont été appréhendés à partir d'une triangulation des différentes sources de données.

Bien que les Nehirowisiwok assistent aux tables GIRT dans plusieurs autres régions, notamment les Laurentides et le Saguenay-Lac-Saint-Jean, l'analyse a été limitée à deux régions par souci de temps et de ressources. Linformation recueillie permet tout de même de porter un certain regard sur la participation nehirowisiw dans les tables GIRT des autres régions puisque les participants rencontrés sont à peu près les mêmes. Par contre, il serait imprudent d'étendre les résultats de la recherche à l'ensemble de la participation autochtone dans les processus de concertation au Québec. Plus particulièrement, la participation de la Société en commandite Scierie Opitciwan a été relativement peu documentée, nous empêchant ainsi de dresser une analyse plus fine de leur contribution à la table GIRT 026-51. Par ailleurs, les comptes rendus ne permettaient pas d'identifier qui intervenait, sauf à quelques occasions. Enfin, tel que mentionné en amont, le fait d'avoir examiné les impacts de la participation nehirowisiw du seul point de vue des participants constitue une autre limite de l'étude.

\section{LES TABLES GIRT ET LA PLANIFICATION FORESTIÈRE}

Les tables GIRT ont été mises en place dans le but explicite d'assurer la collaboration des utilisateurs du territoire dans la planification forestière. Celle-ci se réalise à 
une échelle territoriale locale, soit celle de l'unité d'aménagement forestier (UA) ${ }^{3}$, qui est représentée par le chiffre que l'on retrouve dans le nom de la table (p. ex. Table GIRT « 026-51»). Le $1^{\text {er }}$ avril 2013, le nombre total d'UA au Québec était de 71. La superficie des UA varie énormément $\left(148 \mathrm{~km}^{2}\right.$ à $\left.24447 \mathrm{~km}^{2}\right)$. Selon la nouvelle loi, il peut y avoir une table GIRT pour chaque UA, mais dans les faits, il arrive qu'une seule table soit créée pour représenter les utilisateurs du territoire de plus d'une seule UA. C'est le cas par exemple de la CRNNT de Lanaudière qui a opté pour la création d'une seule table, soit la Table GIRT 062 (UAF 062-51 et 062-52).

Dans le cadre du nouveau régime forestier, il existe deux principaux types de plans d'aménagement forestier ${ }^{4}$, le Plan d'aménagement forestier intégré tactique (PAFIT) et le Plan d'aménagement forestier intégré opérationnel (PAFIO). Les directions générales régionales (DGR) du MFFPQ sont responsables de la réalisation des plans tandis que les Conférences régionales des élus (CRE) ont confié aux CRRNT le mandat d'implanter les tables GIRT et d'assurer leur fonctionnement.

Le PAFIT s'étend sur une période de cinq ans et " présente les objectifs d'aménagement durable des forêts ainsi que la stratégie d'aménagement forestier retenue pour assurer le respect des possibilités forestières et atteindre ces objectifs » (MRNQ 2013). Une des premières étapes consiste à faire un portrait des écarts entre la forêt «naturelle » et la forêt « actuelle » en vue d'un aménagement écosystémique. Le PAFIT doit tenir compte des « préoccupations sociales, économiques et environnementales» dont la formulation est confiée aux tables GIRT. Une fois le PAFIT élaboré, il est alors soumis en consultation publique sous la coordination des CRE et avec l'encadrement des DGR. De son côté, le Ministère consulte les « communautés autochtones affectées par la planification forestière afin de connaître leurs préoccupations relatives aux effets que pourraient avoir les activités planifiées sur leurs activités exercées à des fins domestiques, rituelles ou sociales et les accommode, s'il y a lieu » (L.R.Q. c. A-18.1). Une fois les consultations terminées, le Ministre approuve le plan et le rend aussitôt public.

Le PAFIO établit les zones et les secteurs d'intervention où sont planifiées la récolte de bois et les activités d'aménagement comme les travaux sylvicoles non commerciaux (p. ex. reboisement) ainsi que la construction et l'entretien des chemins (MRNQ 2013). On peut distinguer dans ces plans les zones d'intervention « potentielles » des secteurs d'intervention « finaux ». Le MFFPQ distingue les secteurs d'interventions avec et sans " mesures d'harmonisation ». Celles-ci sont définies par l'État comme étant un moyen « de concilier l'aménagement forestier avec les autres activités pratiquées en forêt »(MFFPQ, s.d.). Le PAFIO peut être réalisé avec la collaboration des tables GIRT et doit être soumis en consultation publique auprès de la population en général ainsi que des communautés autochtones. Encore une fois, c'est le Ministre qui approuve le plan et qui le rend public.
La présente étude porte sur la participation nehirowisiw aux tables GIRT de 2010 à 2013. Cela correspond à la période où les DGR ont élaboré les tout premiers PAFI dans le cadre du nouveau régime forestier. Par ailleurs, la confection des PAFI doit respecter la Stratégie d'aménagement durable des forêts et le Règlement sur l'aménagement durable des forêts, mais puisque ceux-ci n'étaient pas adoptés au moment de réaliser les PAFI, les DGR ont utilisé l'ancienne stratégie et l'actuel Règlement sur les normes d'intervention dans les forêts du domaine de l'État pour élaborer les PAFI (comptes rendus des rencontres des Tables GIRT 062, 026-51, 041-51, 042-51, 043-51 et 043-52).

\section{MANDAT, COMPOSITION ET FONCTIONNEMENT DES TABLES GIRT}

Tel que défini à l'article 55 de la Loi sur l'aménagement durable du territoire forestier (LATDF), le mandat des tables GIRT est « d'assurer une prise en compte des intérêts et des préoccupations des personnes et organismes concernés par les activités d'aménagement forestier planifiées, de fixer des objectifs locaux d'aménagement durable des forêts et de convenir des mesures d'harmonisation des usages» (L.R.Q. c. A-18.1). Le guide élaboré par MFFPQ afin d'orienter la mise en place des tables GIRT précise qu'elles « ont pour mandat de collaborer avec la DGR désignée à l'élaboration des PAFIT et des PAFIO » (Desrosiers et al. 2010 : 7). La CRE de la Mauricie (CREM) reprend à peu près la même définition que celle présentée dans la LADTF :

[...] assurer une prise en compte des intérêts et des préoccupations des personnes, des organismes ayant des mandats, des actions ou des droits sur le territoire et étant concernés par les activités d'aménagement forestier planifiées, de fixer des objectifs locaux d'aménagement durable des forêts et de convenir de certaines mesures d'harmonisation des usages. (CREM $2013: 7$ )

Si le mandat des tables GIRT est délimité par la loi, il semble en aller autrement en ce qui concerne leur composition et leurs règles de fonctionnement. Toujours selon la LADTF, « [leur] composition et [leur] fonctionnement, y compris [leur] modes de règlement des différends, relèvent des organismes régionaux responsables de [leur] mise en place » (L.R.Q. c. A-18.1, article 55). La LADTF indique seulement que les CRE doivent obligatoirement inviter les groupes suivants à participer aux travaux des tables GIRT :

- les communautés autochtones représentées par leur conseil de bande;

- les municipalités régionales de comté, incluant les communautés métropolitaines;

- les bénéficiaires d'une garantie d'approvisionnement;

- les personnes et organismes gestionnaires de zones d'exploitation contrôlée :

- les personnes et organismes autorisés à organiser des activités, à fournir des services ou à exploiter un commerce dans une réserve faunique;

- les titulaires de permis de pourvoirie;

- les titulaires de permis de culture et d'exploitation d'érablière à des fins acéricoles; 
- les titulaires de permis pour la récolte de bois aux fins d'approvisionner une usine de transformation du bois;

- les locataires d'une terre à des fins agricoles;

- les titulaires de permis de piégeage détenant un bail de droits exclusifs de piégeage;

- les conseils régionaux de l'environnement.

(L.R.Q. C. A-18.1, article 55)

Le recrutement des membres des tables GIRT se réalise à travers un processus de nomination (CREM 2013). Les CRE réunissent les intervenants de la région sous forme de forum ou d'assemblée d'utilisateurs et procèdent à des élections. Le membre, une fois élu, détient la responsabilité d'agir à titre de personne ressource sur l'utilisation et le développement des ressources et du territoire, de représenter les intérêts particuliers de son secteur (faune, eau, entreprises sylvicoles, bénéficiaires de garanties d'approvisionnement, autochtones, etc.) et de collaborer à l'identification des « mesures d'harmonisation ». La durée de son mandat est limitée. Par exemple, les membres des tables GIRT de la Mauricie et de Lanaudière sont nommés pour une durée de trois ans (CREM 2013; CRRNTL 2014). Ce processus de nomination ne s'applique pas aux communautés autochtones où ce sont les conseils de bande qui nomment leur représentant, tout comme les municipalités locales. On peut distinguer les membres délégués et leurs substituts qui peuvent prendre part au processus. Des observateurs peuvent agir à titre de personnes ressources ou d'experts (CRE, MFFPQ, consultants), mais ne peuvent pas prendre part à l'élaboration des mesures. Enfin, on retrouve les organisateurs (coordonnateur, président, animateur, etc.) qui assurent le fonctionnement des tables GIRT : organisation des rencontres, préparation des ordres du jour, convocation des membres, comptes rendus des rencontres, etc.

Les tables GIRT ne sont pas décisionnelles. Elles ont pour objectif d'amener les membres à contribuer aux processus de planification forestière en formulant des objectifs locaux d'aménagement et des mesures d'harmonisation générales. La table adopte des recommandations par consensus autant que possible, et ce sont les CRE qui entérinent les mesures formulées (notes d'entretiens). Certaines tables utilisent le vote lorsque le consensus ne peut pas être atteint. Des processus de règlement de différends ont aussi été mis en place. Dans certains cas, les membres de la table peuvent déléguer à un tiers la tâche de trancher parmi les propositions qui n'ont pas fait l'objet d'un consensus. Dans d'autres cas, c'est la DGR du MFFPQ qui tranche en dernier recours (CREM 2013). Dans la région de Lanaudière, la CRRNT propose au MFFPQ la « décision finale $»^{5}$ à retenir. Enfin, certaines tables ont adopté des règles éthiques pour encadrer la participation des membres, par exemple " arriver à l'heure aux réunions »,
Tableau 1

yant participé aux rencontres des tables IRT de la Mauricie et de la Lanaudière de 2010 à 2013

Tables GIRT MAURICIE LANAUDIËRE

\begin{tabular}{|c|c|c|c|c|c|}
$026-51$ & $041-51$ & $042-51$ & $043-51$ & $043-52$ & 062 \\
\hline
\end{tabular}

de Wemotaci

\begin{tabular}{|c|c|c|c|c|c} 
& & $\checkmark$ & $\checkmark$ & $\checkmark$ & \\
\hline$\checkmark$ & $\checkmark$ & & $\checkmark$ & $\checkmark$ & $\checkmark$ \\
\hline$\checkmark$ & & & $\checkmark$ & & \\
\hline
\end{tabular}

* Un chef de territoire semble avoir participé à l'une des rencontres de cette table, mais puisqu'il n'est pas un membre officiel de la table il n'a pas été inscrit au tableau.

« critiquer les idées et non les personnes », adopter une « attitude de coopération plutôt que de confrontation », etc. (CREM 2013).

\section{LA PARTICIPATION NEHIROWISIW AUX TABLES GIRT}

La présentation des résultats sur la participation nehirowisiw aux tables GIRT débute par un portrait des participants suivie de la fréquence de leur présence aux rencontres et de leur représentativité au sein des tables GIRT. Par la suite, le rôle des participants nehirowisiwok est discuté ainsi que les impacts de leur contribution. La présentation se poursuit avec les principaux obstacles à cette participation et se termine avec les mouvances de leurs pratiques participatives et l'identification des facteurs susceptibles d'expliquer les variances observées.

\section{Profil et représentativité DES PARTICIPANTS NEHIROWISIWOK}

Le tableau 1 présente les organisations nehirowisiwok qui sont représentées et qui ont participé aux rencontres des tables GIRT durant la période de novembre 2010 à novembre 2013. Les principaux participants sont les trois conseils de bande ainsi que la Société en commandite Scierie Opitciwan. On remarque que, parmi les tables à l'étude, c'est à la Table GIRT 043-51 que l'on retrouve le plus grand nombre d'organisations nehirowisiwok participantes (3) et que c'est le Conseil des Atikamekw de Manawan qui assiste au plus grand nombre de tables parmi celles à l'étude (4 tables sur 6).

Ce sont les employés des conseils de bande (Centre de ressources territoriales, Bureau de gestion du territoire, etc.) qui assistent aux rencontres des tables GIRT. Toutefois, la présence d'un chef de territoire en tant qu'observateur a été relevée lors d'une rencontre de la Table 043-52 (rencontre du 2011-09-28); celle d'un membre élu du Conseil des Atikamekw d'Opitciwan a aussi été notée à la première rencontre conjointe des Tables 043-51 et 026-51 le 9 novembre 2010. La moitié des membres qui représentent des organisations nehirowisiwok et qui prennent part aux rencontres des tables GIRT ne sont pas des Nehirowisiwok; ils sont employés notamment à titre d'ingénieurs ou de techniciens forestiers. 
Tableau 2

Représentativité des participants nehirowisiw aux rencontres des tables GIRT de Lanaudière (062) et de la Mauricie (026-51, 041-51, 043-51, 042-51 et 043-52)

\begin{tabular}{|c|c|}
\hline TABLES GIRT & ReprÉsEntATIVITÉ NeHIROWISIWOK/MEMBres \\
\hline Table GIRT 062 & 1 sur $17(6 \%)$ \\
\hline Table GIRT 026-51 & 2 sur $10(20 \%)$ \\
\hline Table GIRT 041-51 & 1 sur $15(7 \%)$ \\
\hline Table GIRT 043-51 & 3 sur $17(18 \%)$ \\
\hline Tables GIRT $042-51$ et $043-52$ & 1 sur $15(7 \%)$ \\
\hline
\end{tabular}

La représentativité des membres nehirowisiwok varie d'une table à l'autre. Dans l'ensemble, les résultats indiquent qu'ils sont minoritaires et qu'ils représentent de 6 à $20 \%$ des membres qui siègent au sein des tables GIRT (voir tableau 2). Les Tables GIRT 042-51 et 043-52 tiennent habituellement des rencontres conjointes puisque les membres sont presque identiques. Cela explique pourquoi la représentativité est la même pour ces deux tables. Il faut noter que les résultats présentés dans le tableau ne tiennent pas compte de la présence du substitut qui accompagne parfois le représentant nehirowisiw. Il faut également préciser qu'en pratique, la représentativité fluctue en fonction du nombre de membres délégués nehirowisiwok et non nehirowisiwok présents aux rencontres. À partir de la table GIRT affectée à l'unité d'aménagement forestier 043-51, voici un exemple qui illustre la représentativité des divers utilisateurs et intervenants : aire faunique communautaire (1); bénéficiaires de garantie d'approvisionnement (3); communautés autochtones (3); environnement et eau (1); entreprise en travaux sylvicoles (1); MRC (1); pourvoirie (1); tourisme et récréation (1); trappeur (1); villégiateur (1); communautés locales (vacant); autres détenteurs (vacant). À cela s'ajoutent les représentants de la CRE (2-3) et du MFFPQ (2-3) à titre de membres non votants.

\section{FrÉQUence de LA PARTICIPATION NEHIROWISIW}

Dans la région de la Mauricie, les tables GIRT ont tenu au total 46 rencontres entre le 9 novembre 2010 (première rencontre) et le 7 novembre 2013 (voir tableau 3). Les résultats démontrent que les Nehirowisiwok ont participé en moyenne à sept rencontres sur dix. Lorsqu'ils sont présents, les données indiquent qu'il y a en moyenne au moins deux représentants nehirowisiwok (trois rencontres sur quatre). Ce qui pourrait expliquer cette tendance serait le fait que 25 rencontres sur 42 étaient des rencontres conjointes réunissant les membres de deux tables et plus (voir Fortier, en préparation, pour plus de détails). Du côté de la région lanaudoise, douze rencontres ont été tenues au total au cours de la période à l'étude. La première rencontre s'est déroulée le 30 novembre 2010 et la dernière a eu lieu le 23 octobre 2013. Les Nehirowisiwok ont participé à huit rencontres sur douze (67\%). La plupart du temps, il y avait un seul représentant (six rencontres sur huit). Ainsi, de 2010 à 2013, tant du côté des tables GIRT de la Mauricie que de Lanaudière, la fréquence de la participation nehirowisiw aux rencontres est demeurée, de manière générale, plutôt continue (sept rencontres sur dix). Toutefois, il y a eu un événement important qui semble avoir eu un effet « temporaire » sur la fréquence de participation, et nous y reviendrons un peu plus loin.

\section{RÔLE DES PARTICIPANTS NEHIROWISIWOK}

Les représentants nehirowisiwok qui assistent aux réunions des tables GIRT affirment d'emblée ne pas avoir reçu de mandat de leurs organisations pour appuyer les recommandations et les mesures élaborées par les membres délégués (notes d'entretiens). Par conséquent, ils agissent plutôt à titre de membres délégués observateurs. Ils interviennent à l'occasion afin d'informer les autres membres de la table à propos des préoccupations que la communauté pourrait avoir sur certains sujets traités lors des réunions. Les intervenants non nehirowisiwok présents aux tables GIRT semblent apprécier ce type d'intervention de la part des représentants nehirowisiwok:

Nous on aime ça quand ils viennent aux rencontres parce qu'ils amènent toujours des choses même s'ils ne se positionnent pas. [...] Parfois ils vont poser des questions puis là, il y a des gens du ministère ou de l'industrie ou d'autres qui réalisent un bout de leur réalité qu'ils n'ont jamais eu l'occasion de se faire expliquer. Des fois [un tel] explique: "Mais non, là, nous autres ça fonctionne de même puis c'est telle affaire. Puis quand on parle de telle chose, bien c'est le chef de famille, ou bien quand on parle de telle autre, c'est telle personne qui doit être au courant ou qui doit avoir de l'information. » (Représentant de la CRE)

Toutefois, on ressent, dans d'autres témoignages, que certaines attentes demeurent inassouvies à l'égard de la contribution des Nehirowisiwok aux tables GIRT, incluant notamment les travaux des CRRNT :

Quand ils sont là et qu'on a des discussions, ils ne participent pas beaucoup. [...] On ne les comprend pas parce qu'on ne les connaît pas. Moi c'est ce que je pense. Ils pourraient apporter leurs connaissances. Ça, ça serait incroyable, mais ils ne sont pas très présents et quand ils parlent c'est pour dire : "On veut de I'emploi. » (Représentant de la CRE)

Les résultats ont révélé que si les Nehirowisiwok ne participent pas directement à l'adoption des recommandations élaborées par les membres des tables GIRT, ils font tout de même connaître leur point de vue par d'autres moyens. Par exemple, la communauté de Manawan a pris connaissance à un moment donné d'un dossier en assistant à une rencontre d'une table GIRT; il s'agissait alors de l'adoption d'un protocole de fermeture des chemins par suite des travaux forestiers afin notamment de mieux contrôler l'accès aux « territoires structurés » (ZEC, pourvoiries, réserves fauniques):

\footnotetext{
Au niveau de la table GIRT, quand on fait une planification forestière, parfois on crée de nouveaux chemins. Puis ça crée des problèmes surtout pour les pourvoiries [et] les ZEC parce que ça crée parfois une nouvelle ouverture au territoire. Puis là, il y a plus de risque de braconnage, plus de difficulté à contrôler l'accès et tout. On voulait pouvoir proposer des fermetures de chemins mais présentement la fermeture de chemins c'est très complexe
} 
comme processus. Puis [le Ministère] lui-même nous a dit à une réunion de la Commission I'année passée : "[Telle région] a adopté un processus accéléré, vous pouvez copier là-dessus et on va l'accepter. » [...] Finalement, ça a été adopté en avril, mais au moment où on l'a adopté le Ministère nous a dit: " Finalement on a eu des discussions avec les Atikamekw, ils sont contre les fermetures de chemins. " Alors on l'a adopté mais il n'est pas applicable. [...] Dans le fond, ils nous ont dit : " C'est correct, mais assurez-vous que les Atikamekw sont d'accord. » Mais dans [telle région], ils n'ont pas dit oui encore à aucune fermeture de chemin. (Représentant de la CRE)

Ainsi, il appert que ce n'est pas à la table GIRT (ni à la CRRNT) que Manawan a exprimé son point de vue mais plutôt dans un « processus en parallèle » de la table GIRT (et de la CRRNT). Autrement dit, c'est plutôt en ligne directe avec l'État que la communauté fait connaître ses préoccupations vis-à-vis de la planification forestière, incluant notamment la question des « chemins forestiers » :

En fait, les Atikamekw considèrent qu'ils ont juste à négocier avec le Ministère, avec le gouvernement fédéral et provincial. En fait, quand on parle de ce qui est nos dossiers «planification forestière " puis tout ça, chaque fois qu'on avait, mettons, une décision, à la table GIRT, qui était consensuelle, [l'ingénieur forestier de la communauté] disait : "N'oubliez pas que moi, c'est comme si je ne votais pas parce qu'on a notre consultation en parallèle. » On l'appelle de "nation à nation » ou " en parallèle. » (Représentant de la CRE)

D'autre part, certains participants nehirowisiwok rencontrés ont aussi remarqué des différences entre leur rôle au sein des tables GIRT et celui d'autres nations autochtones telles que les Innus et les Hurons:

II y a des communautés qui sont plus avancées dans les tables GIRT. Ils ne viennent pas comme personnes participantes, ils viennent là comme personnes ressources au même niveau que le ministère. [...] Ils ne viennent pas comme participants. Ils viennent comme « ressources » de la table. (Participant nehirowisiw)

La table, finalement, amène ses conclusions, ses recommandations au ministère. Dans ce cas-là, elle les amène au ministère et à la communauté [autochtone]. Ils sont à ce niveau-là, eux autres. Ils ne sont pas identifiés comme un intervenant sur le territoire qui se mêle aux dix-huit autres puis aux vingt-deux autres. (Consultant du conseil de bande)

Par ailleurs, l'un des témoins rencontrés a révélé que certains groupes autochtones ne participaient pas aux tables GIRT (Participant nehirowisiw). Ainsi, les représentants nehirowisiwok qui assistent aux rencontres sont en mesure de se comparer aux autres groupes autochtones et de constater ainsi les différents rôles que peuvent jouer les communautés autochtones au sein d'un processus de concertation comme celui des tables GIRT.

Une dernière chose qui mérite d'être soulignée ici est celle de la participation nehirowisiw aux comités ad hoc et aux groupes de travail sur des enjeux plus techniques et spécifiques tels que la qualité des habitats fauniques, la
Tableau 3

ation nehirowisiw aux tables GIRT

et 043-52) de 2010 à 2013

NOMBRE DE RENCONTRES

FRÉQUENCE AUX RENCONTRES

\begin{tabular}{l|l}
\hline 12 rencontres & $8 / 12(66,67 \%)$ \\
\hline
\end{tabular}

46 rencontres ( 25 ren-

contres conjointes *

$32 / 46(69,57 \%)$

58 rencontres

$40 / 58(69,97 \%)$

ndiquent que certaines tables GIRT tenaient des rencontres conjointes lorsque la

délimitation des aires protégées et le développement de la villégiature. Selon certains témoignages recueillis, c'est souvent dans ces sous-comités de travail que les représentants des Nehirowisiwok contribuent davantage:

[...] ce qui les rejoint le plus [...] c'est les groupes de travail sur un sujet spécifique comme dans les groupes de travail sur les aires protégées. J'ai eu par moment des gens de chacune des trois communautés ou parfois j'en avais deux. C'est rare les rencontres où je n'en ai eu aucune [...] aussi on a fait le plan de développement du réservoir Gouin [...] puis ça interpelle tout de suite Opitciwan. Alors ils ont participé. Ils étaient à toutes les rencontres puis ils se sont positionnés puis ils ont amené leurs points de vue [...]. Mais ça c'est un mandat très spécifique. On parlait du développement de toute la villégiature et des pourvoiries au réservoir Gouin. Là, ils participent, ils sont actifs puis ils amènent des choses. (Représentant de la CRE)

En somme et pour l'heure actuelle, les CRE et leurs CRRNT laissent la porte ouverte aux communautés nehirowisiwok à titre de membres votants (table GIRT) tandis que, dans les faits, les membres nehirowisiwok agissent plutôt à titre d'observateurs et de rapporteurs auprès de la communauté qu'ils ou elles représentent.

\section{RETOMBÉES ET EFFETS DE LA PARTICIPATION NEHIROWISIW}

À la lumière des résultats présentés en amont, les raisons pour lesquelles les Nehirowisiwok assistent aux tables GIRT demeurent somme toute à expliquer. En effet, ils n'ont pas le mandat d'appuyer les recommandations des tables $^{6}$ et ils ont recours à d'autres mécanismes « en parallèle » pour se faire entendre. Pourquoi alors participer si ce n'est pas pour prendre part à l'élaboration des objectifs locaux devant orienter la planification et l'aménagement du territoire et des ressources? Cela semble s'expliquer à première vue par les diverses retombées indirectes qu'ils en retirent.

Premièrement, un des témoignages recueillis a révélé que les subventions gouvernementales reçues par les autorités nehirowisiwok contiennent des conditions obligatoires comme celle d'assister aux tables GIRT : « [...] le financement qu'on a eu c'était... il était comme conditionnel à ce qu'on y participe. Bon, on va y participer. » (Consultant du conseil de bande) Il s'agit dans ce cas-ci d'une référence au Programme de mise en valeur du milieu forestier du MRNQ (volet II) et qui fut remplacé en 
juillet 2013 par le Programme de développement régional et forestier.

Du côté de la communauté de Manawan, la participation aux tables GIRT s'inscrit aussi dans une démarche plus globale conforme à une entente conclue avec le gouvernement du Québec en 2003 (notes d'entretiens). Elle s'imprègne ainsi d'une volonté de la communauté de réaliser des partenariats avec le milieu régional afin d'assurer notamment son développement social et économique: «Pour eux, je sens que ce qu'ils veulent, justement, c'est qu'on contribue à leur développement puis qu'on les aide à se développer, puis à avoir une meilleure qualité de vie. » (Représentant de la CRE) Certains témoignages ont révélé que c'est aussi une autre façon de " protéger le territoire » mais aussi de mieux informer la communauté sur les rouages du nouveau régime forestier :

[...] on a pris la décision d'assister juste pour être en mesure d'informer la communauté sur comment se déroule, comment s'implante le régime. [...] Puis on a l'avantage de le voir dans une, deux, trois, quatre régions différentes. (Consultant du conseil de bande)

[...] notre principal intérêt de participer à ça c'est de bien connaître l'application, la nouvelle façon de gérer la forêt pour pouvoir bien renseigner la communauté ou les organismes qui veulent s'en occuper de façon à trouver des moyens de mieux intervenir pour tout le temps la même chose, pour la personne qui utilise le territoire. (Consultant du conseil de bande)

Les rencontres des tables GIRT représentent aussi une occasion pour les membres d'apprendre à se connaître. Par exemple, l'animateur des tables de la Mauricie demandait aux membres d'exprimer leurs attentes lors de la première rencontre. De plus, certains membres des tables GIRT interagissaient pour la première fois avec un Nehirowisiw. Autrement dit, les membres non nehirowisiwok bénéficient aussi de la présence nehirowisiw en développant une meilleure connaissance de leur culture et de leurs préoccupations. Certains témoignages des Nehirowisiwok ont également souligné le potentiel de créer de nouvelles alliances: " Les autres utilisateurs de la forêt, ils ont les mêmes préoccupations que nous autres. On peut trouver des alliés là-dedans. » (Participant nehirowisiw)

Enfin, certains témoignages ont précisé que les rencontres des tables GIRT permettaient de développer et d'entretenir un « lien de confiance », et même de favoriser des contacts supplémentaires entre les personnes qui sont engagées dans diverses démarches en parallèle. Cela inclut, entre autres, ceux qui participent à plusieurs tables GIRT ainsi qu'aux consultations du MFFPQ menées directement auprès des communautés nehirowisiwok:

On a des rencontres officielles d'harmonisation, mais le fait qu'on se voyait aussi aux tables GIRT, ça créait chaque fois des opportunités de rencontres puis de discussions à l'extérieur de la rencontre d'harmonisation d'un chantier où, là, j'avais des discussions complémentaires d'un chantier ou en amont d'un autre qui s'en venait. (Représentant du MFFPQ)

Pour résumer, les principales retombées de la participation nehirowisiw aux tables GIRT sont l'accès aux programmes d'aide financière de l'État, le développement et le maintien de relations avec d'autres groupes d'acteurs. Leur participation permet aussi de faire connaître leurs préoccupations aux membres non autochtones. De plus, les tables GIRT ont représenté une source d'information capitale pour les représentants des communautés afin de mieux comprendre les changements engendrés par la mise en place du nouveau régime forestier.

\section{Quelques ObStacles À LA PARTICIPATION}

Bien que les Nehirowisiwok aient participé à la majorité des rencontres des tables GIRT pendant la période à l'étude, leur participation a rencontré aussi quelques obstacles. On retrouve premièrement la distance entre la localité des communautés et le lieu géographique des rencontres des tables GIRT. Souvent les représentants doivent parcourir quelques centaines de kilomètres pour assister aux rencontres. Ensuite, malgré le financement octroyé pour l'aide à la concertation, les ressources des communautés sont limitées et suffisent souvent à peine à couvrir le salaire de leurs représentants ainsi que leurs frais de déplacements. Le roulement du personnel à l'intérieur des organisations nehirowisiwok peut aussi ralentir la participation et engendrer de l'absentéisme lors des rencontres (notes d'entretiens). De plus, les représentants nehirowisiwok n'ont pas le mandat de se positionner officiellement sur les recommandations adoptées par la table GIRT. Ce qui représente un frein important à leur contribution. Enfin, puisque les processus de consultation menés en parallèle par l'État permettent déjà aux Nehirowisiwok de donner leur point de vue sur la planification forestière, cela peut également affaiblir leur collaboration aux tables GIRT.

\section{MOUVANCES DE LA PARTICIPATION NEHIROWISIW}

Les pratiques participatives des Nehirowisiwok semblent avoir peu évolué depuis la mise en place des tables GIRT en 2010. Certains ajustements apportés aux tables (p. ex. l'ajout de nouveaux sièges) ne paraissent pas avoir eu d'impact significatif sur leurs pratiques. Par contre, le blocus des chemins forestiers par les Nehirowisiwok en Haute-Mauricie durant l'été de 2012 (CNW 2012), suivi du déclenchement des négociations bipartites avec le gouvernement provincial, a eu un impact important sur leur participation aux tables GIRT dans la région de la Mauricie - où elle a chuté drastiquement (notes d'entretiens). En effet, d'après les analyses de fréquence de la présence nehirowisiw aux tables GIRT de cette région, si l'on compare le taux de participation aux rencontres avant et après le blocus des Nehirowisiwok lors de l'été 2012, le taux chute de près de la moitié (voir Fortier, en préparation, pour les détails). Enfin, rappelons que certains témoignages ont soulevé que le roulement de personnel au sein des conseils de bande pouvait avoir un effet sur la participation en créant temporairement un certain absentéisme (notes d'entretiens).

\section{COOPTATION, RÉSISTANCE ET COLLABORATION DANS LES tABLES GIRT DE LA MAURICIE ET DE LANAUDIĖRE}

Au cours de l'année 2010, les tables GIRT sont apparues un peu partout au Québec. Définies comme des processus de concertation à travers lesquels les divers 
utilisateurs d'un territoire délimité déterminent conjointement les objectifs locaux d'aménagement (i.e. à l'échelle des unités d'aménagement forestier), elles ont été implantées avant tout afin de répondre aux exigences légales de la LADTF (L.R.Q. C. A-18.1, article 55). Dans un contexte où l'on reconnaît maintenant que les Premières Nations ne peuvent plus être considérées comme simples «intervenants » ou « utilisateurs » du territoire (p. ex. pourvoirie, municipalité, etc.), cet article avait pour objectif d'évaluer la nature et les effets de la participation nehirowisiw tout en prenant soin d'en décrire le cadre.

Les résultats ont révélé que les principaux participants étaient les membres des équipes de gestion du territoire des conseils de bande et la Société en commandite Scierie Opitciwan. Leur présence demeure plutôt continue et elle n'influence peu ou pas les objectifs d'aménagement locaux élaborés par les tables GIRT dans le cadre de la planification forestière. En effet, les participants nehirowisiwok affirment ne pas détenir de mandat de leur communauté pour participer à titre de partie prenante et, par conséquent, ils jouent essentiellement un rôle d'observateurs et de rapporteurs. Un élément important qui expliquerait cette position serait l'existence des processus de consultation menés en parallèle par l'État et qui permettent déjà aux Nehirowisiwok de donner leur point de vue sur les plans d'aménagement forestier au sein d'une relation « de nation à nation ». Cela diffère largement du rôle qui leur est offert à titre de simples «intervenants » au sein des tables GIRT, rôle qui demeure assez marginal (p. ex. 1/17). Cette "marginalisation» semble également se refléter au sein d'autres structures régionales telles que les CRE et les CRRNT. Selon Grammond, c'est bien plus qu'une marginalisation «numérique » mais également une « logique de gouvernance » qui «assimile les peuples autochtones à des municipalités locales » et où « les droits particuliers et le statut spécial des peuples autochtones [...] se retrouvent noyés dans une structure dont la mission se limite à la consultation et à la concertation entre les partenaires "locaux" ( Grammond 2009: 952).

La présence plutôt soutenue des Nehirowisiwok aux rencontres des tables GIRT s'explique, selon nous, d'abord par une forme de cooptation de leur participation. En effet, le fait d'obliger les communautés à assister aux tables GIRT pour avoir accès à certains programmes de l'État représente un facteur clé de cette participation. Bien que celle-ci soit en partie "cooptée », les représentants agissent seulement à titre de d'observateurs, ce qui constitue à nos yeux une forme de résistance à la cooptation puisqu'ils refusent ainsi délibérément de jouer le rôle que l'on attend d'eux.

Les résultats ont démontré que la participation nehirowisiw a peu évolué entre 2010 et 2013. Par contre, le déclenchement du blocus des chemins forestiers par les Nehirowisiwok à l'été 2012, qui a été suivi par les négociations bipartites avec le gouvernement du Québec, a eu un effet significatif mais temporaire sur la fréquence de leur présence aux rencontres des tables GIRT, surtout dans la région de la Mauricie. Cela illustre que leur participation peut être influencée par des facteurs exogènes au processus tout comme d'autres études l'ont déjà illustré (Fortier 2008; Ansell et Gash 2008).

Il a aussi été démontré que les communautés autochtones (nehirowisiwok et autres) ne s'engagent pas de façon identique aux processus de concertation comme ceux des tables GIRT. Les témoignages des Nehirowisiwok ont permis d'entrevoir que certains groupes refusent d'assister aux tables GIRT alors que d'autres communautés interviennent au même titre que les CRE ou les DGR du MFFPQ (p. ex. les Innus de Mashteuiatsh ou « Pekuakamiulnuatsh Takuhikan »). Enfin, d'autres communautés collaborent à titre de membres délégués pouvant voter tout comme les autres membres. Par conséquent, on peut raisonnablement soutenir que la participation varie d'une nation à l'autre et d'une communauté à l'autre, et que l'étude des processus de concertation « multipartenaires » représente un champ de recherche potentiellement très prometteur pour des études comparatives sur la participation autochtone à la gestion forestière au Québec.

En somme, les résultats ont démontré que les Nehirowisiwok reconnaissent les limites de ce qui peut être réalisé dans les tables GIRT. Par conséquent, ils ont décidé d'adopter le rôle d'observateurs au lieu de partie prenante, tout en se gardant le droit d'intervenir occasionnellement. Ainsi, les tables GIRT ne constituent pas pour les Nehirowisiwok le lieu où l'on détermine la gestion du territoire. Elles ne sont pas non plus un espace qui leur permet d'exercer ou de faire reconnaître leurs droits en ce qui concerne le territoire. Néanmoins, elles sont un espace qui permet d'établir et de maintenir des liens avec les autres utilisateurs, d'apprendre à se connaître et de s'informer concernant les activités sur le territoire. Ce qui confirme d'ailleurs les propos de Smith et Bombay (1995) qui ont été rapportés en introduction, ainsi que les conclusions d'autres études sur les effets des processus collaboratifs dans le contexte de la gestion des ressources et du territoire (Ansell et Gash 2008; Gunton et Day 2003; Innes et Booher 1999; Selin et Chavez 1995; Gray 1989). Dans ce contexte, il semble que les Nehirowisiwok ont fait le choix stratégique de ne pas trop s'investir dans ces tables. Ils ne se prêtent pas au jeu de l'État qui voit plutôt dans les tables un moyen de réconcilier les intérêts divergents. Bref, les Nehirowisiwok sont parvenus à trouver un moyen de participer aux tables tout en poursuivant leurs propres aspirations.

\section{Notes}

1. Cet article constitue l'un des chapitres d'une recherche doctorale (Fortier, en préparation) portant sur l'évolution de la participation des Atikamekw Nehirowisiwok dans la planification forestière de 1990 à 2013. La recherche a été menée en collaboration avec le Conseil de la Nation atikamekw (CNA). Toutefois, les opinions, conclusions et recommandations exprimées dans le présent article sont celles des auteurs et ne doivent pas être interprétées comme étant celles du CNA.

2. Au moment de l'implantation des tables GIRT, c'était le ministère des Ressources naturelles et de la Faune du Québec 
(MRNFQ). Plus récemment, l'élection d'un nouveau gouvernement libéral en avril 2014 a mené au scindement du ministère pour former le ministère de l'Énergie et des Ressources naturelles et le ministère des Forêts, de la Faune et des Parcs (MFFPQ). Afin d'alléger le texte, l'acronyme MFFPQ est utilisé pour faire référence à ce ministère (division « Forêt »), quels que soient les noms qu'il a portés au fil du temps.

3. Pour plus d'informations à ce sujet : <http://www.mffp.gouv. qc.ca>.

4. Il existe aussi les plans d'aménagement spéciaux pour certains contextes précis : feux de forêt, épidémies, etc.

5. <http://www.crrnt-lanaudiere.com/concertation/table-degestion-integree-des-ressources-et-du-territoire/mission-etmandat/> (consulté le 8 mai 2014).

6. À l'exception peut-être du représentant de la Société en commandite Scierie Opitciwan.

\section{Remerciements}

Les auteurs tiennent à remercier tout d'abord les participants à l'étude ainsi que Sylvie Poirier et les lecteurs anonymes pour leurs commentaires. Nous désirons également souligner le soutien financier de l'Institut Hydro-Québec en environnement, développement et société, du Réseau DIALOG, de la Fondation Desjardins, du Conseil de recherches en sciences humaines du Canada et du Fonds de recherche du Québec - Société et culture. De plus, l'auteur principal tient à affirmer toute sa reconnaissance envers le Conseil de la Nation atikamekw (CNA) et les Conseils des Atikamekw de Manawan, de Wemotaci et d'Opitciwan qui ont collaboré tout au long du projet de recherche. Enfin, un merci tout particulier à Gérald Ottawa, Samuel Castonguay et Richard Boivin pour leur guidance bienveillante et leurs conseils.

\section{Ouvrages cités}

ANSELL, Chris, et Alison GASH, 2008 : «Collaborative governance in theory and practice ». Journal of Public Administration and Theory Advances 18(4) : 543-571.

BURQUEL, Jean-Maurice, 1992 : « La table de concertation sur le développement durable lance un projet pilote en milieu forestier ». L'Écho de La Tuque, 16 août : 12.

CARLSSON, Lars, et Fikret BERKES, 2005: « Co-management: concepts and methodological implications ». Journal of Environmental Management 75 : 65-76.

CCMF (Conseil canadien des ministres des forêts), 1995 : Définir la gestion durable des forêts: Une approche canadienne aux critères et indicateurs. Conseil canadien des ministres des forêts, Ottawa (Ontario).

—, 2003: Définir l'aménagement forestier durable au Canada: Critères et indicateurs. Conseil canadien des ministres des forêts, Ottawa (Ontario).

CONLEY, Alexander, et Margaret A. MOOTE, 2003 : « Evaluating Collaborative Natural Resource Management». Society and Natural Resources 16 : 371-386.

CNW, 2012 : «Blocus d'opérations forestières : la Nation atikamekw exige le respect de ses droits ». Canada News Wire.

CÔTÉ, Marc-André, et Luc BOUTHILLIER, 2002 : « Assessing the effect of public involvement processes in forest management in Quebec ». Forest Policy and Economics 4 : 213-225.

CREM (Conférence régionale des élus de la Mauricie), 2013: Règles et modalités de fonctionnement des tables de gestion intégrée des ressources et du territoire (TGIRT). Version amendée le 5 décembre 2013, Conférence régionale des élus de la Mauricie.

CRRNTL (Commission régionale des ressources naturelles et du territoire de la Lanaudière), 2014: Règles et modalités de fonctionnement. Table locale de gestion intégrée des ressources et du territoire (table GIRT) 062. Commission régionale des ressources naturelles et du territoire de la Lanaudière.

CRRNTM (Commission régionale des ressources naturelles et du territoire de la Mauricie), 2012 : Table locale de gestion intégrée des ressources et du territoire (TGIRT) de la Mauricie. Évaluation annuelle 20011-2012. Commission régionale des ressources naturelles et du territoire de la Mauricie.

DESROSIERS, R., S. LEFEBVRE, P. MUNOZ et J. PAQUET, 2010 : Guide sur la gestion intégrée des ressources et du territoire: son application dans l'élaboration des plans d'aménagement forestier intégré. Ministère des Ressources naturelles et de la Faune du Québec.

DIDUCK, Alan P., et A. John SINCLAIR, 2002 : « Public Involvement in Environmental Assessment: the Case of Nonparticipant ». Environmental Management 29(4) : 578-588.

FEIT, Harvey A., 2005 : « Re-cognizing co-management as cogovernance: visions and histories of conservation at James Bay ». Anthropologica 47(2) : 267-288.

FEIT, Harvey A., et Robert BEAULIEU, 2001 : «Voices from a disappearing forest », in Colin H. Scott (dir.), Aboriginal Autonomy and Development in Northern Quebec and Labrador: 119-148. UBC Press, Vancouver.

FORTIER, Jean-François, 2008 : «Lîle René-Levasseur, œil du Québec ou de Nitassinan? Une lecture sociologique des enjeux contemporains de la gouvernance territoriale autochtone ». Aspects sociologiques 15(1): 73-92.

-, [en préparation] : Une gouvernance forestière en mouvance: Regards exploratoires sur l'évolution des rapports entre les Nehirowisiwok ("Atikamekw »), l'État et l'industrie forestière dans le cadre de la planification et de l'aménagement forestier (de 1990 à 2013). Thèse de doctorat, Faculté des sciences sociales, Université Laval.

GÉLINAS, Claude, 2002 : « La création des réserves atikamekw (1895-1950), ou quand l'Indien était vraiment un Indien ». Recherches amérindiennes au Québec 32(2): 35-46.

-, 2003: Entre l'assommoir et le godendart. Les Atikamekw et la conquête du Moyen-Nord québécois 1870-1940. Septentrion, Québec.

GOSSELIN, Marie 2004: Les modèles de cogestion du territoire. Institut de développement durable des Premières Nations du Québec et du Labrador, Québec.

GRAMMOND, Sébastien, 2009: «La gouvernance territoriale au Québec entre régionalisation et participation des peuples autochtones ». Revue canadienne de science politique 24(4) : 939-956.

GRAY, Barbara, 1989 : Collaborating: Finding Common Ground for Multiparty Problems. Jossey-Bass, San Francisco.

GUNTON, Thomas I., et J.C DAY, 2003 : « The Theory and Practice of Collaborative Planning in Resource and Environmental Management ». Environments 31(2): 5-19.

HOUDE, Nicolas, 2012 : "Experimenting with what will become our traditions": Adaptive co-management as a bridge to an Atikamekw Nehirowisiw post-treaty world in Nitaskinan, Canada. Thèse de doctorat, département de géographie, Université McGill, Montréal.

HOWLETT, M., J. RAYNER et C. TOLLEFSON, 2009 : «From government to governance in forest planning? Lessons from the case of the British Columbia Great Bear Rainforest Initiative ». Forest Policy and Economics 11 : 383-391.

INNES, Judith E., et David E. BOOHER, 1999: «Consensus building as Role Playing and Bricolage: Towards a Theory of Collaborative Planning ». Journal of the American Planning Association 65(1) : 9-26.

LECLERC, Édith, et Caroline ANDREW, 2013: «Les tables de concertation de gestion intégrée des ressources forestières: est-ce que les GIR gouvernent? » in Guy Chiasson et Judith 
Leclerc (dir.), La gouvernance locale des forêts publiques québécoises. Une avenue de développement des régions périphériques? 127-146. Presses de l'Université du Québec.

Loi sur l'aménagement durable du territoire forestier, L.R.Q., c. A-18.1.

MARTINEAU-DELISLE, Catherine 2013 : La participation publique et la gestion des forêts au Québec: Changement de gouvernance, impacts des pratiques et profil des participants. Thèse de doctorat, département de sociologie, Université Laval.

McGREGOR, Deborah, 2006 : «La participation autochtone à l'aménagement durable des forêts en Ontario: des avancées vers la coopération ». Recherches amérindiennes au Québec 36(2-3) : 61-70.

—, 2011 : «Aboriginal/non-Aboriginal Relations and Sustainable Forest Management in Canada: The Influence of the Royal Commission on Aboriginal Peoples ». Journal of Environmental Management $92:$ 300-310.

McNEIL, Kent, 1997 : " The Meaning of Aboriginal Title», in Michael Asch (dir.), Aboriginal and Treaty Rights in Canada: Essays on Law, Equality and Respect for Difference: 135-154. UBC Press, Vancouver.

MFFPQ (ministère des Forêts, de la Faune et des Parcs du Québec), s.d. : Glossaire forestier. <http://glossaire-forestier.mffp.gouv. qc.ca/terme. aspx? $\mathrm{id}=159 \& \mathrm{pub}=\mathrm{O}>$

MRNQ (ministère des Ressources naturelles du Québec), 2013 : Planification forestière. Ministère des Ressources naturelles du Québec.

NADASDY, Paul, 2003 : Hunters and bureaucrats: Power, knowledge, and aboriginal-State relations in the Southwest Yukon. UBC Press, Vancouver.

NADEAU, S., C. MARTINEAU-DELISLE et J.-F. FORTIER, 2004 : La participation publique à la gestion forestière par l'entremise des comités : portrait de la situation dans quelques régions du Québec. Rapport préparé pour la Commission d'étude sur la gestion de la forêt publique québécoise.

NATCHER, David, et Suzan DAVIS, 2007 : « Rethinking Devolution: Challenges for Aboriginal Resource Management in the Yukon Territory ». Society and Natural Resources $20: 271-279$.

Nation Haïda c. C.-B. [2004] 3 R.C.S. 511.

NATIONS UNIES (Division Développement durable), 1992a : Rapport de la Conférence des Nations Unies sur l'Environnement et le Développement. <http://www.un.org/documents/ga/conf151/ french/aconf15126-lannexlf.htm>

—, 1992b : Déclaration de principes, non juridiquement contraignante mais faisant autorité, pour un consensus mondial sur la gestion, la conservation et l'exploitation. <http://www.un.org/french/events/ rio92/aconf15126vol3f.htm>

—, 1992c : Agenda 21. <http://www.un.org/french/ga/special/sids/ agenda21/action 0. htm $>$

PINKERTON, Evelyn, 2003 : « Toward specificity in complexity: Understanding co-management from a social perspective », in D. C. Wilson, J. R. Nielson et P. Degnbol (dir), The fisheries co-management experience: Accomplishments, challenges and prospects : 61-77. Dordrecht, Pays-Bas, Kluwer Acadamic Publishers.

PLUMMER, Ryan, et John FITZGIBBON, 2004 : « Some observations on the terminology in co-operative environmental management ». Journal of Environmental Management 70 : 63-72.

POIRIER, Sylvie, 2000 : «Contemporanéités autochtones, territoires et (post)colonialisme. Réflexions sur des exemples canadiens et australiens ». Anthropologie et Sociétés 24(1) : 137-155.

QUÉBEC, Gouvernement du, 2002 : Entente concernant une nouvelle relation entre le gouvernement du Québec et les Cris du Québec. Bibliothèque et Archives nationales du Québec, Québec.
—, 2008 : Guide intérimaire en matière de consultation des communautés autochtones. Bibliothèque et Archives nationales du Québec, Québec.

ROBILLARD, Alexandre, 2014 : «Les Atikamekw déclarent leur indépendance ». La Tribune, mardi 9 septembre, p. 9.

RODON, Thierry 2003: En partenariat avec l'État: les expériences de cogestion des Autochtones du Canada. Les Presses de l'Université Laval.

ROY, Simon N., 2010 : « L'étude de cas », in Benoît Gauthier (dir.), Recherche sociale. De la problématique à la collecte de données: 199-225. Presses de l'Université du Québec.

SAA (Secrétariat aux Affaires autochtones), 1998 : Convention de la Baie-James et du Nord québécois et conventions supplémentaires. Secrétariat aux Affaires autochtones, Québec.

SAVARD, Rémi, 1981 : Le Sol américain : propriété privée ou terremère... L'en-deçà et l'au-delà des conflits territoriaux entre autochtones et Blancs au Canada. L'Hexagone, Montréal.

SELIN, Steve, et Deborah CHAVEZ, 1995: «Developping a Collaborative Model for Environmental Planning and Management». Environmental Management 19(2) : 189-195.

SIMARD, Jean-Jacques, 2003 : La Réduction. L'Autochtone inventé et les Amérindiens d'aujourd'hui. Septentrion, Québec.

SMITH, Peggy, et Harry BOMBAY, 1995 : Aboriginal Participation in Forest Management: Not Just Another Stakeholder. National Aboriginal Forestry Association.

SPAEDER, Joseph J., et Harvey FEIT, 2005 : « Cogestion et collectivités autochtones: entraves et rapprochements dans la gestion décentralisée des ressources ». Anthropologica 47(2): 155-163

STEVENSON, Marc, et David C. NATCHER, 2010: Planning co-existence: Aboriginal issues in forest and land use planning. CCI Press, Edmonton.

STEVENSON, Marc, et Jim WEBB, 2003 : « Just another stakeholder? First Nations and sustainable forest management in Canada's boreal forest. Chapter 3 », in P.J. Burton, C. Messier, D.W. Smith et W.L. Adamowicz (dir.), Towards Sustainable Management of the Boreal Forest : 65-112. NRC Research Press, Ottawa.

TAKEDA, Louise, et Inge RØPKE, 2010 : « Power and contestation in collaborative ecosystem-based management: The case of Haida Gwaii ». Ecological Economics 70 : 178-188.

THÉBERGE, Delphine, 2012 : Rencontre des acteurs et des imaginaires à travers un processus de dialogue. Le cas du Projet pilote d'aménagement écosystémique de la Réserve faunique des Laurentides. Mémoire de maîtrise, Faculté des sciences sociales, Université Laval, Québec.

VON DER PORTEN, Suzanne, et Robert C. DE LOË, 2014 : « How collaborative approaches to environmental problem solving view indigenous peoples: A systematic review ». Society and Natural Resources 27(10): 1-17.

WYATT, Stephen, 2004 : Co-existence of Atikamekw and industrial forestry paradigms: Occupation and management of forestlands in the St-Maurice river basin, Québec. Thèse de doctorat, Faculté de foresterie et de géomatique, Université Laval, Québec.

_, 2006: «Si eux le font, pourquoi pas nous? La quête des Atikamekw de Wemotaci pour un rôle dans la foresterie dans le Nitaskinan ». Recherches amérindiennes au Québec 36(2-3) : 9-18.

WYATT, S., J.-F. FORTIER et C. MARTINEAU-DELISLE, 2010 : « First Nations' Involvement in Forest Governance: The Place for Distinct Processes ». The Forestry Chronicle 86(6) : 730-741. 